\title{
Studies of carrier dynamics in unintentionally doped gallium nitride bandtail states
}

\author{
Chi-Kuang Sun, ${ }^{a)}$ Jian-Chin Liang, and Xiang-Yang Yu \\ Department of Electrical Engineering and Graduate Institute of Electro-Optical Engineering, \\ National Taiwan University, Taipei 10617, Taiwan, Republic of China \\ Stacia Keller, Umesh K. Mishra, and Steven P. DenBaars \\ Department of Electrical and Computer Engineering, University of California, Santa Barbara, \\ California 93106
}

(Received 31 August 2000; accepted for publication 20 February 2001)

\begin{abstract}
Ultrafast carrier dynamics of bandtail states in an unintentionally doped gallium nitride sample was investigated using femtosecond transient transmission measurements. The transient responses of shallow bandtail states resemble those of above band gap extended states. The transient responses of the deep bandtail states are, on the other hand dominated by carrier transfer into the lower energy states through phonon assisted tunneling suggesting that the deep bandtail states are localized states. (C) 2001 American Institute of Physics.
\end{abstract}

[DOI: $10.1063 / 1.1366650]$

Gallium nitride (GaN)-based semiconductors have recently attracted a lot of attention for their applications as light emitters in the blue to UV wavelength range. ${ }^{1}$ Although a considerable amount of research has been conducted on the optical properties of these materials, there is still much left unknown about the optical processes associated with radiative recombination and stimulated emission (SE) mechanisms. Many reports suggested that the bandtail states play an important role in these mechanisms. ${ }^{2-7}$ Osiński et al. reported the evidence of the involvement of bandtail states in the radiative recombination in blue and green singlequantum well light-emitting diodes. ${ }^{3}$ Chichibu et al. indicated that the stimulated emission of InGaN multiplequantum wells (MQWs) might be originated from localized bandtail states just below the mobility edge. ${ }^{2,4}$ Optically pumped SE experiments by Schmidt et al. reported that gain originating from localized states in InGaN MQWs was responsible for the observed SE. ${ }^{6,7}$ However, detail carrier dynamics of bandtail states in these materials are not clear yet.

In order to understand the carrier dynamics of bandtail states in a GaN-based semiconductor, we have performed carrier dynamic studies on an unintentionally doped $\mathrm{GaN}$ thin film using standard femtosecond pump-probe techniques. The GaN thin film was grown by metalorganic chemical vapor deposition on $c$-plane sapphire in an atmospheric pressure reactor. After annealing the substrate at $1050^{\circ} \mathrm{C}$, a $525-\AA$-thick nucleation layer was deposited at $600{ }^{\circ} \mathrm{C} .{ }^{8}$ The temperature was then raised to $1080{ }^{\circ} \mathrm{C}$ to grow an unintentionally doped $\mathrm{GaN}$ layer of $5 \mu \mathrm{m}$ thickness. The $n$-type carrier concentration on the $\mathrm{GaN}$ bulk layer was $\sim 1 \times 10^{16} \mathrm{~cm}^{-3}$. The crystal structure was wurzite. ${ }^{8}$ Figure 1 shows the measured room temperature photoluminescence (PL) spectrum photoexcited using a continuous-wave $\mathrm{He}-\mathrm{Cd}$ pump laser at $325 \mathrm{~nm}$ wavelength. The observed peak at 3.42 $\mathrm{eV}(362 \mathrm{~nm})$ corresponds to band edge transition. Two different emission tails below the band gap can be observed.

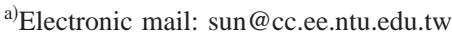

One is around $3.35 \mathrm{eV}(370 \mathrm{~nm})$ and the other gradually decays below $3.32 \mathrm{eV}(373 \mathrm{~nm})$. It is similar to the results previously reported by Qui et al. ${ }^{9}$ They are referred to as shallow bandtail and deep bandtail, respectively. The measured absorption spectrum on the sample was also shown in Fig. 1. There are two absorption tails, an abrupt tail close to the band edge between 3.32 and $3.40 \mathrm{eV}$ and a gradually decaying one deeper into the gap below $3.32 \mathrm{eV}$, in agreement with the measured PL. The absorption spectrum indicates that the shallow bandtail emission in this sample is below the exciton emissions that should be located around $3.4 \mathrm{eV}$ at room temperature. ${ }^{10}$

Transient transmission pump-probe experiments were performed on these below band edge bandtail transitions using a 150 fs Ti: sapphire laser. The infrared laser output was focused into a $500-\mu \mathrm{m}$-thick beta barium borate (BBO) crystal to produce frequency-doubled UV light tunable between 360 and $400 \mathrm{~nm}(3.44-3.10 \mathrm{eV})$, corresponding to the vicinity of band edge transition and below band gap transition. After the BBO crystal, the infrared pulses were removed using a color glass filter. Measurements were performed using a standard transmission type pump-probe geometry. One tenth of the UV beam used as the probe beam was reflected by a beamsplitter and passed through a variable delay stage. The rest of the UV power passed through the beamsplitter was used as the pump beam. The polarization of the probe beam was rotated $90^{\circ}$ with a half-wave plate. The probe beam was vertically polarized while the pump beam maintained horizontally polarized. The pump and probe pulses were focused into the sample using an objective lens with a focal length of $1 \mathrm{~cm}$. We estimated the spot size from the transmission ratio passing through a pinhole of $10 \mu \mathrm{m}$ diameter. The estimated focal spot diameter was $14 \mu \mathrm{m}$. The transmitted UV pump was rejected using an iris and a polarizer so that only the transmitted probe signal was detected. The pump beam was chopped at $1 \mathrm{kHz}$ and the detected probe signal was measured as a function of the temporal delay between the pump and probe using a lock-in amplifier. 


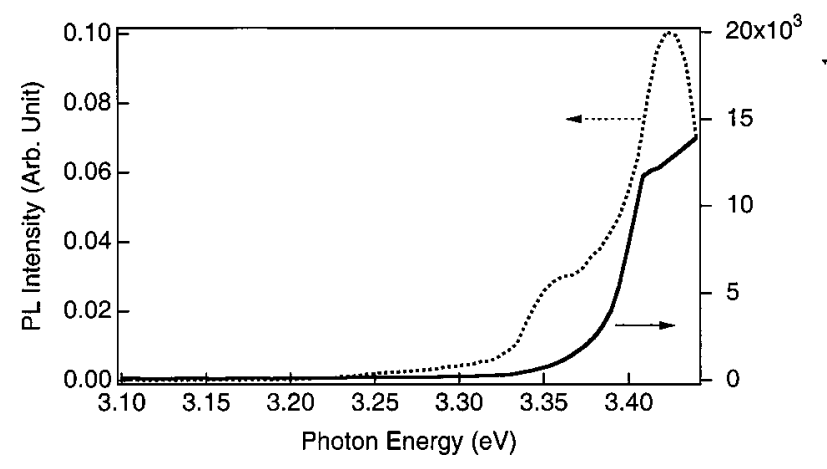

FIG. 1. Photoluminescence spectrum (dashed line, left axis) and absorption spectrum (solid line, right axis) for bulk GaN at room temperature. Two emission tails can be observed.

The transient transmission responses $\Delta T / T$ as a function of probe delay for shallow bandtail state region at pump/ probe photon energies of $3.37 \mathrm{eV}(367.5 \mathrm{~nm})$ and $3.34 \mathrm{eV}$ $(371 \mathrm{~nm})$ are shown in Figs. 2(a) and 2(b), respectively. Both traces show an absorption saturation signal with a fast relaxation process followed by a flat plateau after several picoseconds. By using convolution fits, exponential decay time constant on the order of $500 \mathrm{fs}$ can be obtained for the initial fast relaxation process. These traces mimic the above band gap transient transmission traces obtained using the same technique previously in $\mathrm{InGaN}^{11}$ and extensive studies in both $\mathrm{InGaN}^{11}$ and bulk $\mathrm{GaN}^{12-14}$ have assigned this 500 fs component to external carrier thermalization processes (the equilibrium process between carrier system and phonon system). Above band gap transient transmission measurement in the same sample showed same behaviors as these shallow bandtail traces. Compared with previous femtosecond studies around the band edge of bulk GaN, ${ }^{12-14}$ it suggests that this fast relaxation component might be contributed from the external thermalization processes of photoexcited carriers.

It is noticed that the signal size of $\Delta T / T$ was proportional to the change of absorption coefficient $\Delta \alpha$, which was proportional to the density of states (DOS) under a fixed pump fluence. With a fixed pump fluence of $60 \mu \mathrm{J} / \mathrm{cm}^{2}$, the amplitude of $\Delta T / T$ decreased from $4.2 \times 10^{-2}$ at a wavelength of $367.5 \mathrm{~nm}$ to $4.7 \times 10^{-3}$ at a wavelength of $371 \mathrm{~nm}$. This reflects that the DOS is lower at lower energy side of shallow bandtail states. While we tuned our experimental wavelength from 367.5 to $371 \mathrm{~nm}$, the observed flat plateau was gradually transferred from a negative step response into a positive step response. We attributed the negative step response to the band gap renormalization effect ${ }^{15}$ that was also reported previously in InGaN. ${ }^{11}$ As we gradually move our excitation wavelength away from the band edge, the band gap renormalization signal disappeared and the bandfilling signal was restored, which is responsible for the positive step response observed in Fig. 2(b).

As we moved our excitation wavelength into the deep bandtail region below $3.32 \mathrm{eV}$, distinct transient responses were observed. In Figs. 3(a) and 3(b), we show the measured transient transmission responses for the pump/probe photon energies of $3.28 \mathrm{eV}(377 \mathrm{~nm})$ and $3.10 \mathrm{eV}(400 \mathrm{~nm})$, respectively. The pump fluence was fixed at $60 \mu \mathrm{J} / \mathrm{cm}^{2}$. In contrast to the responses of pump/probe photon energies greater than $3.35 \mathrm{eV}$, a sharp negative two-photon absorption signal ${ }^{16,17}$
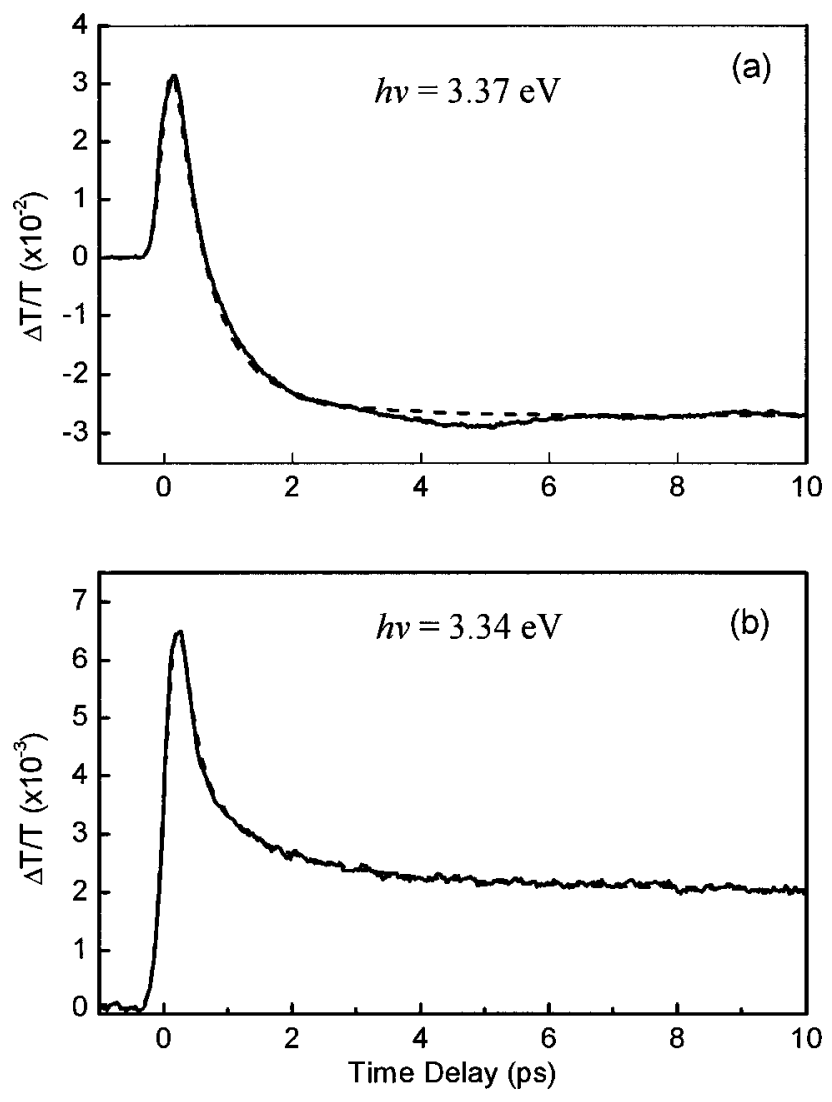

FIG. 2. Measured transient transmission response for carriers photoexcited in the shallow bandtail states. The pump-probe photon energies are (a) 3.37 and (b) $3.34 \mathrm{eV}$. The dotted lines are convolution fits.

followed by a positive slowly exponentially decayed component was observed. No fast thermalization process can be found. The disappearance of the two-photon absorption peak in Fig. 2 was attributed to the existence of the large positive external thermalization components and the absorption saturation effect due to higher density of states in shallow bandtail states. ${ }^{18}$ Previous $Z$-scan study ${ }^{18}$ on the same sample showed a transition between two-photon absorption and absorption saturation around $374 \mathrm{~nm}(3.31 \mathrm{eV})$, in agreement with our transient transmission measurement. Excellent convolution fits (dotted lines in Fig. 3) can be obtained by using a negative delta function and a positive single-exponential decay function without any step functions. The time constants for the slowly decaying components can thus be derived. The time constant of this component is on the order of several tens of picoseconds while the measured time constant was found to increase with decreased photon energy. Time constants of 27, 33, and 59 ps were obtained for photon energies of $3.316,3.294$, and $3.256 \mathrm{eV}$, respectively. This behavior can be understood as the effect of phonon-assisted tunneling, which occurs for localized states. The decay time of the carrier is not only due to radiative recombination but also due to the carrier transfer process into the deeper but isolated tail states through the assistance of acoustic phonons. ${ }^{19}$ With the effect of phonon assisted tunneling, the carrier decay time $\tau(E)$ can be described as a function of the photon energy $E$ as

$$
\tau(E)=\frac{\tau_{r}}{1+\exp \left({ }^{\left(E-E_{m e}\right)} / E_{0}\right)},
$$



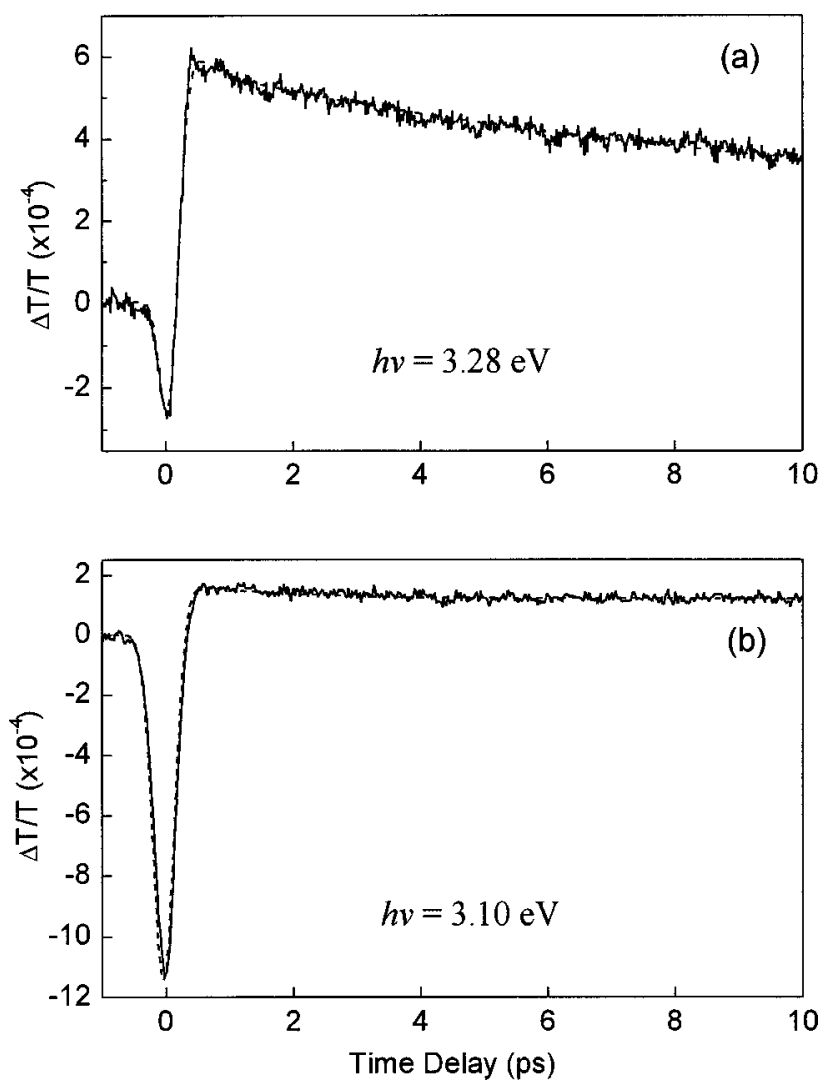

FIG. 3. Measured transient transmission response for carriers photoexcited in the deep bandtail states. The pump-probe photon energies are (a) 3.28 and (b) $3.10 \mathrm{eV}$. The dotted lines are convolution fits.

where $\tau_{r}$ is the radiative lifetime, $E_{\mathrm{me}}$ is the energy for which the decay time equals the transfer time, and $E_{0}$ is the characteristic energy for the DOS. According to Eq. (1), the carrier decay time will increase with decreased photon energy, agreeing with our observations. We have used Eq. (1) to fit the decay time measured from pump-probe experiment. Excellent fitting can be obtained, indicating the localized nature of the deep bandtail states. The obtained fitting is shown in Fig. 4 with $\tau_{r}=180 \mathrm{ps}, E_{\mathrm{me}}=3.22 \mathrm{eV}$, and $E_{0}=52 \mathrm{meV}$. Our results indicate a bandtail carrier radiative lifetime on the order of $180 \mathrm{ps}$ for this specific GaN sample. It is also interesting to notice that the $\Delta T / T$ amplitudes of the slowly decaying components decrease from $6.8 \times 10^{-4}$ at $377 \mathrm{~nm}$ to $1.1 \times 10^{-4}$ at $400 \mathrm{~nm}$ with a fixed pump fluence, reflecting the continuously decreased DOS toward the lower energy side of deep bandtail.

In summary, we have studied the bandtail states in an unintentionally doped GaN thin film. We measured the PL and absorption spectra at room temperature and two different bandtails were observed. Standard transmission pump-probe experiments were performed. Dynamic behaviors for carriers in shallow bandtail states are found to be dominated by a fast carrier external thermalization process with a time constant on the order of $500 \mathrm{fs}$, which is with the same characteristic of above band gap extended states. Dynamic behaviors for carriers in deep bandtail states are, on the other hand, dominated by a slow single-exponential decay without any fast

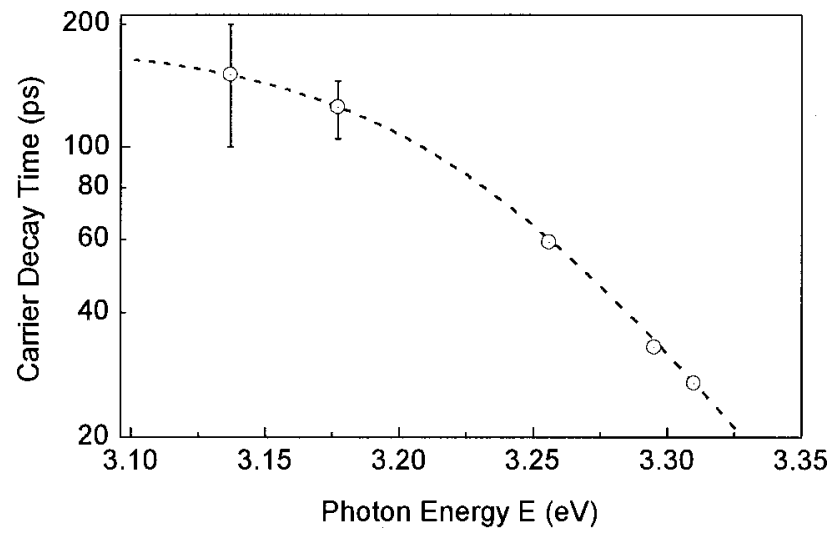

FIG. 4. Measured carrier lifetimes (open circles) in the deep localized states. Longer lifetime was observed for lower energy states. Dotted line is a fitting curve with Eq. (1).

thermalization behavior. The carrier behavior in deep bandtail states agrees well with a model of phonon-assisted tunneling, indicating the localized state nature of the deep bandtail states. The radiative carrier lifetime in deep localized states was on the order of $180 \mathrm{ps}$.

The authors would like to acknowledge the stimulated scientific discussion with Professor S. C. Shen. This research is supported by National Science Council of Taiwan, R.O.C. under Grant No. 89-2112-M-002-082.

${ }^{1}$ S. Nakamura and G. Fasol, The Blue Laser Diode: GaN Based Light Emitters and Lasers (Springer, Berlin, 1997).

${ }^{2}$ S. Chichibu, T. Azuhata, T. Sota, and S. Nakamura, Appl. Phys. Lett. 69, 4188 (1996).

${ }^{3}$ P. G. Eliseev, P. Perlin, J. Lee, and M. Osiński, Appl. Phys. Lett. 71, 569 (1997).

${ }^{4}$ Y. Narukawa, Y. Kawakami, M. Funato, S. Fujita, S. Fujita, and S. Nakamura, Appl. Phys. Lett. 70, 981 (1997).

${ }^{5}$ A. Satake, Y. Masumoto, T. Miyajima, T. Asatsuma, F. Nakamura, and M. Ikeda, Phys. Rev. B 57, R2041 (1998).

${ }^{6}$ T. J. Schmidt, Y.-H. Cho, G. H. Gainer, J. J. Song, S. Keller, U. K. Mishra, and S. P. Denbaars, Appl. Phys. Lett. 73, 560 (1998).

${ }^{7}$ T. J. Schmidt, Y.-H. Cho, G. H. Gainer, J. J. Song, S. Keller,U. K. Mishra, and S. P. Denbaars, Appl. Phys. Lett. 73, 1892 (1998).

${ }^{8}$ B. P. Keller, S. Keller, D. Kapolnek, W.-N. Jiang, Y.-F. Wu, H. Masui, H. Wu, B. Heying, J. S. Speck, U. K. Mishra, and S. P. DenBaars, J. Electron. Mater. 24, 1707 (1995).

${ }^{9}$ C. H. Qui, C. Hoggatt, W. Melton, M. W. Leksono, and J. I. Pankove, Appl. Phys. Lett. 66, 2712 (1995).

${ }^{10}$ S. Chichibu, T. Azuhata, T. Sota, H. Amano, and I. Akasaki, Appl. Phys. Lett. 70, 2085 (1997).

${ }^{11}$ C.-K. Sun, F. Vallée, S. Keller, J. E. Bowers, and S. P. DenBaars, Appl. Phys. Lett. 70, 2004 (1997).

${ }^{12}$ C.-K. Sun, Y.-L. Huang, S. Keller, U. K. Mishra, and S. P. DenBaars, Phys. Rev. B 59, 13535 (1999).

${ }^{13}$ H. Ye, G. W. Wicks, and P. M. Fauchet, Appl. Phys. Lett. 74, 711 (1999).

${ }^{14}$ H. Ye, G. W. Wicks, and P. M. Fauchet, Appl. Phys. Lett. 77, 1185 (2000).

${ }^{15}$ Hot Carriers in Semiconductor Nanostructures, Physics and Applications, edited by J. Shah (Academic, Boston, 1992).

${ }^{16}$ K. L. Hall, J. Mark, E. P. Ippen, and G. Eisenstein, Appl. Phys. Lett. 56, 1740 (1990).

${ }^{17}$ C.-K. Sun, J.-C. Liang, J.-C. Wang, F.-J. Kao, S. Keller, M. P. Mack, U. Mishra, and S. P. DenBaars, Appl. Phys. Lett. 76, 439 (2000).

${ }^{18}$ C.-K. Sun, Y.-L. Huang, J.-C. Liang, J.-C. Wang, K.-G. Gan, F.-J. Kao, S. Keller, M. P. Mack, U. Mishra, and S. P. DenBaars, Opt. Quantum Electron. 32, 619 (2000).

${ }^{19}$ C. Gourdon and P. Lavallard, Phys. Status Solidi B 153, 641 (1989). 